\title{
Empirical comparison of microsatellite and SNP markers to estimate introgression in Apis mellifera mellifera
}

\author{
Melanie Parejo, Dora Henriques, M. Alice Pinto, Gabriele Soland-Reckeweg \& \\ Markus Neuditschko
}

To cite this article: Melanie Parejo, Dora Henriques, M. Alice Pinto, Gabriele Soland-Reckeweg \& Markus Neuditschko (2018) Empirical comparison of microsatellite and SNP markers to estimate introgression in Apis mellifera mellifera, Journal of Apicultural Research, 57:4, 504-506, DOI: 10.1080/00218839.2018.1494894

To link to this article: https://doi.org/10.1080/00218839.2018.1494894

\section{+ View supplementary material $๘$}

\section{Published online: 20 Sep 2018.}

Submit your article to this journal $\widetilde{ }$

Џll Article views: 70

View Crossmark data $\nearrow$ 


\title{
NOTES AND COMMENTS
}

\section{Empirical comparison of microsatellite and SNP markers to estimate introgression in Apis mellifera mellifera}

\author{
Melanie Parejo ${ }^{\mathrm{a}, \mathrm{b} *}$, Dora Henriques ${ }^{\mathrm{c}, \mathrm{d}}$, M. Alice Pinto ${ }^{c}$, Gabriele Soland-Reckeweg ${ }^{\mathrm{e}}$ and Markus Neuditschko ${ }^{\mathrm{a}}$ \\ ${ }^{a}$ Agroscope, Swiss Bee Research Centre, Bern, Switzerland; 'Vetsuisse Faculty, Institute of Bee Health, University of Bern, Bern,

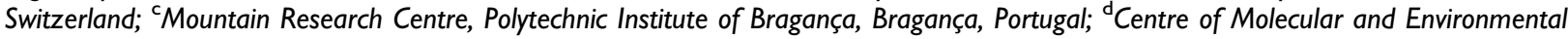 \\ Biology, University of Minho, Braga, Portugal; ${ }^{\mathrm{e}}$ Apigenix, Twann, Switzerland
}

(Received 14 October 2017; accepted 16 May 2018)

\begin{abstract}
The genetic identity of the dark European honey bee, Apis mellifera mellifera is currently under pressure throughout most of its native range due to large scale commercial trade and replacement with honey bees of mainly Eastern European ancestry (C-lineage: Apis mellifera carnica and Apis mellifera ligustica). To counteract this process, numerous conservation efforts for the protection of native honey bees are sprouting across Europe. For the management of such protected areas and conservation breeding purposes, honey bee subspecies have been routinely identified through wing morphology and through DNA-hybrid tests using microsatellite markers. Currently, new methods are evolving including rapid innovations in single-nucleotide polymorphism (SNP) array technology and high-throughput sequencing. Here, we aim to quantify potential marker-specific biases of hybrid tests and give recommendations for applications in honey bee conservation management. Using an empirical dataset, we first assessed the accuracy of a recently developed reduced SNP panel to estimate C-lineage introgression in A. m. mellifera compared to whole-genome sequence (WGS) data. Using another independent data set, we estimated the differences in admixture proportions between the currently applied hybrid test based on microsatellites and the novel SNP test. We demonstrate that the SNP-based test which contains highly ancestry-informative markers is very efficient to estimate genome-wide ancestry. Furthermore, we report discrepancies between microsatellite and SNP-based admixture proportions. For conservation management, we, therefore, recommend the implementation of SNP-based hybrid tests to maintain high genetic variation within the breeding population, while minimizing influence of introduced honey bees.
\end{abstract}

KEYWORDS: Apis mellifera mellifera; introgression; microsatellites; SNPs; conservation

Persisting colony losses of managed honey bees worldwide call for sustainable solutions to protect populations against diverse stressors. The breeding of locally adapted subspecies and ecotypes may prove to be a powerful approach for the long-term sustainability of populations (Meixner et al., 20l0). A recent panEuropean experiment has revealed that native subspecies and local hybrids have longer survivorship (Büchler et al., 2014) and lower pathogen loads (Francis et al., 2014) than introduced colonies. However, recurring importations of commercial breeding stock may interfere with complex genetic traits of locally adapted honey bees (Meixner et al., 20l0). To date, numerous conservation breeding programs and protected areas have been established in Europe to preserve and promote native honey bees (reviewed by De La Rúa, Jaffé, Dall'Olio, Muñoz, \& Serrano, 2009).

In Switzerland, importations and breeding of mainly A. m. carnica and Buckfast bees have cornered the native dark European honey bee, A. m. mellifera, which has led to increasing conservational breeding efforts and the establishment of currently four protected areas. Hybrid colonies in such protected areas and in breeding stock have been routinely identified through wing morphology (Parejo, Wragg, Henriques, Vignal, \& Neuditschko, 2017) and more recently through genetic analysis based on microsatellite markers (Bouga et al., 20ll). However, new hybrid tests are being developed using genome-wide SNPs (Muñoz et al., 2015; Parejo et al., 2016).

The aim of this study was to validate a recently developed SNP-based hybrid test against WGS information and compare ancestry proportions inferred from two different marker types (microsatellites and SNPs) to give recommendations for future management strategies focusing on the conservation of $A$. m. mellifera.

$A$ reduced SNP panel to estimate $C$-lineage introgression in $A$. m. mellifera was recently developed by Henriques et al. (2018) consisting of 117 ancestryinformative SNPs (see Online Supplementary Material for further information). In a first step, this reduced SNP panel was tested in an independent dataset consisting of 31 drone genomes from Switzerland originating from a previous study (Parejo et al., 2016). These individuals were selected to cover a broad range of C-lineage ancestry (see Online Supplementary Material).

\footnotetext{
*Corresponding author. Email: melanie.parejo@alumni.ethz.ch

(4) Supplemental data for this article can be accessed here.
} 


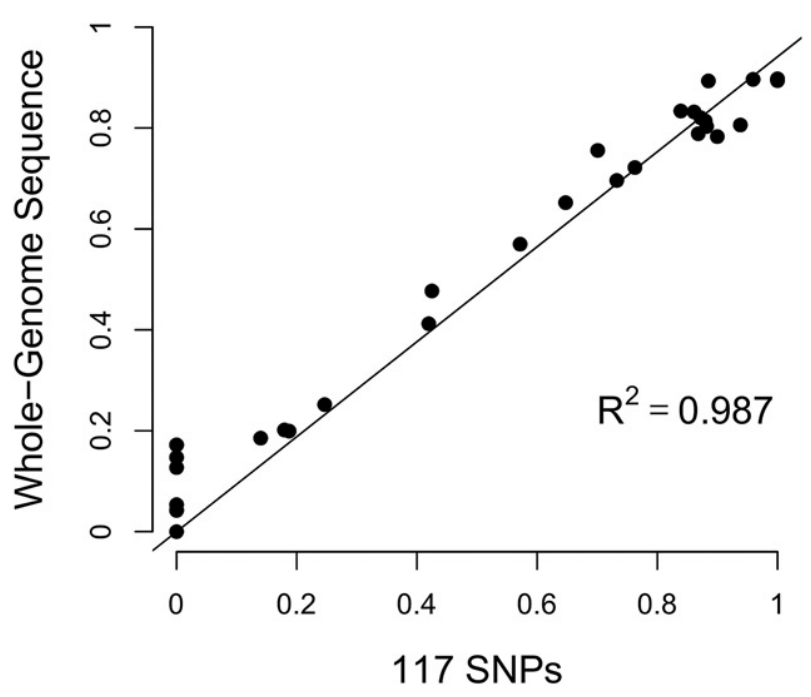

Figure I. Linear regression analysis $(y=0.942 x, p<.00 \mathrm{I})$. Ancestry proportions as estimated by the 117 ancestry-informative SNPs accurately predict genome-wide ancestry (3.077 MSNPs).

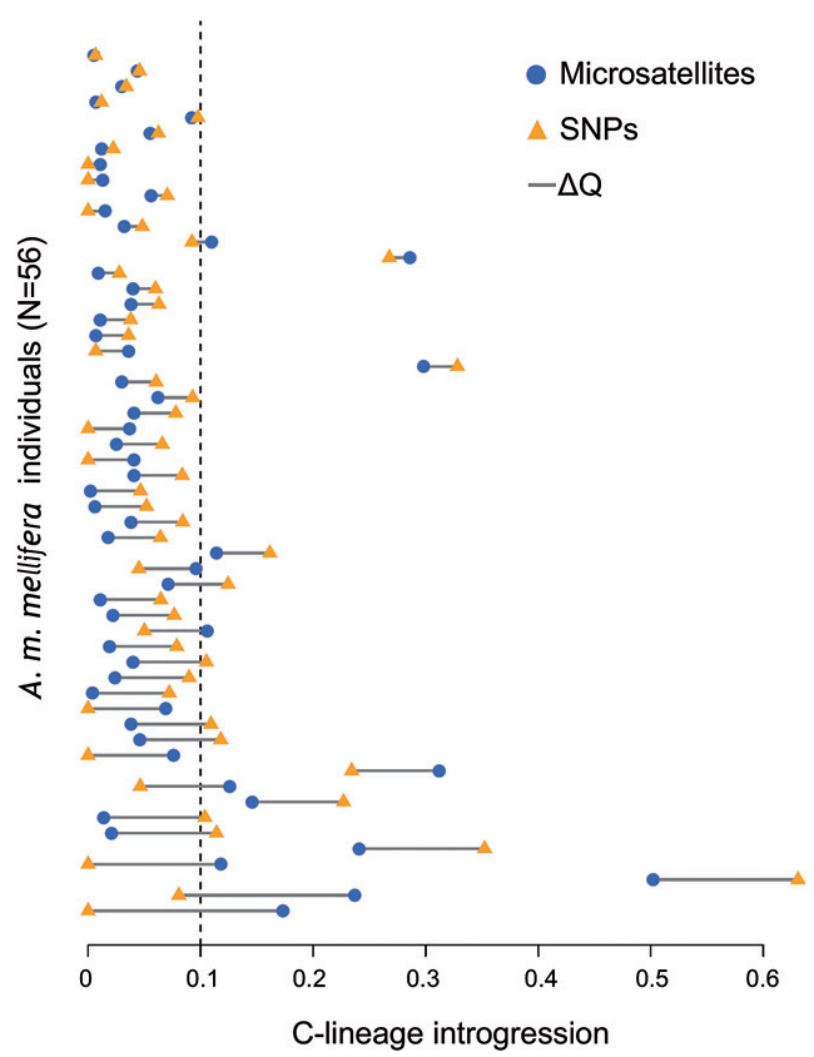

Figure 2. C-lineage proportions estimated by 12 microsatellites (blue circles) and 117 ancestry-informative SNPs (yellow triangles) for 56 A. m. mellifera samples from Switzerland ordered from smallest to largest differences. The $\Delta Q$-values $\left(Q_{S N S^{-}}\right.$ $Q_{\text {Microsatellites }}$ ) are represented by solid grey lines. The dashed black line indicates the 10\% C-lineage introgression threshold currently applied for pure race breeding in Switzerland.

The ancestry proportions estimated using this panel are very highly correlated with those inferred from WGSs (3.077 M SNPs) (Pearson's $r=0.990, \mathrm{df}=29, p<.00 \mathrm{I}$ ) (Figure I), which represents the most accurate ancestry inference. Therefore, this result indicates that the panel containing only II7 SNPs can successfully be applied to accurately estimate ancestry in an independent data set.

The currently employed DNA-hybrid test in Switzerland is based upon 12 microsatellite markers (see supplementary material) (Bouga et al., 20II). To evaluate this test, we used a second independent data set of $56 \mathrm{~A}$. m. mellifera from the Swiss breeding population and protected areas. We then compared ancestry proportions inferred from the 12 microsatellites against the proportions estimated with the 117 SNPs. For this comparison, we calculated the differences in C-lineage introgression $\left(\Delta Q\right.$-value $\left.=Q_{S N P s}-Q_{\text {Microsatellites }}\right)$ estimated using the two different marker types (Figure 2). In general, there was a high congruence between the two estimations $(60 \%$ of samples with less than $5 \%$ difference). However, in 23 out of 56 samples we observed a difference greater than $5 \%$ in the estimation of C-lineage introgression and for five samples even a difference greater than $10 \%$. The currently implemented C-lineage threshold in Switzerland to select an A. m. mellifera colony for the breeding population is $10 \%$. With regard to this threshold, we observed six samples that were erroneously included in the breeding population or protection area even though they would be classified as hybrid based on SNP-markers (Figure 2). We further observed six colonies which were classified as hybrids using microsatellites but not when using SNPs. In this case, $\mathrm{C}$-lineage introgression has been overestimated by microsatellites and pure colonies are excluded which potentially reduce genetic diversity in the breeding population. Possible explanations for the discrepancies include the use of different reference populations and different characteristics of the two marker types (e.g., high mutation rate of microsatellites). In addition, the microsatellites only cover 8 out of 16 honey bee chromosomes, while the SNPs are distributed across all chromosomes. In practice, microsatellites have proved to be a valuable genetic resource for honey bee breeding management due to their cost-effectiveness and flexibility regarding number of samples genotyped. However, our and other results (Muñoz et al., 2017) indicate a lower resolution of random microsatellites compared with highly informative SNPs. Therefore, we recommend implementing SNP-testing to improve conservation management decisions.

In this study, we first validated the recently developed SNP panel for estimation of C-lineage introgression using an independent data set. We show that ancestry proportions using these 117 SNPs are highly correlated with the proportions inferred from WGSs highlighting that sequencing whole-genomes is not required for ancestry inference. Secondly, we compared the results of the SNP panel with the currently applied hybrid test for the breeding management of A. m. mellifera in Switzerland. We noticed that, on average, a high congruence between the two different marker types 
exists. However, we also identified larger discrepancies in few samples which require further investigation. Employing cost-effective and highly accurate molecular tools to estimate introgression in $A . m$. mellifera is increasingly important in conservation management. We demonstrated that the novel SNP-based hybrid test is very accurate compared to WGS data and was successfully applied in the Swiss A. m. mellifera population. Consequently, it is recommended as an improved tool in addition to the currently employed microsatellite test.

\section{Disclosure statement}

No potential conflict of interest was reported by the authors.

\section{Funding}

This work was supported by the Swiss Federal Office for Agriculture FOAG, the Fondation Sur-la-Croix, Basel, and by the 2013-2014 BiodivERsA/FACCE-JPI joint call for research proposals, with the national funders "Fundação para a Ciência e Tecnologia" (Portugal), "Agence Nationale de la Recherche" (France), and "Ministerio de Economía y Competividad" (Spain).

\section{References}

Bouga, M., Alaux, C., Bienkowska, M., Büchler, R., Carreck, N. L., Cauia, E., ... Wilde, J. (20II). A review of methods for discrimination of honey bee populations as applied to European beekeeping. Journal of Apicultural Research, 50(I), 5I-84. doi: I0.3896/IBRA.I.50.I.06

Büchler, R., Costa, C., Hatjina, F., Andonov, S., Meixner, M. D., Le Conte, Y., ... Wilde, J. (20I4). The influence of genetic origin and its interaction with environmental effects on the survival of Apis Mellifera L. colonies in Europe.
Journal of Apicultural Research, 53(2), 205-2I4. doi:10.3896/ IBRA. I.53.2.03

De La Rúa, P., Jaffé, R., Dall'Olio, R., Muñoz, I., \& Serrano, J. (2009). Biodiversity, conservation and current threats to European honey bees. Apidologie, 40(3), 263-284.

Francis, R. M., Amiri, E., Meixner, M. D., Kryger, P., Gajda, A., Andonov, S., ... Wilde, J. (20/4). Effect of genotype and environment on parasite and pathogen levels in one apiary A case study. Journal of Apicultural Research, 53(2), 230-232. doi:I0.3896/IBRA.I.53.2.14

Henriques, D., Browne, K. A., Barnett, M. W., Parejo, M., Kryger, P., Freeman, T., ... Pinto, M. A. (20/8). High sample throughput genotyping for estimating $\mathrm{C}$-lineage introgression in the dark honey bee: An accurate and costeffective SNP-based tool. Scientific Reports, 8(I), 8552.

Meixner, M. D., Costa, C., Kryger, P., Hatjina, F., Bouga, M., Ivanova, E., \& Büchler, R. (20I0). Conserving diversity and vitality for honey bee breeding. Journal of Apicultural Research, 49(I), 85-92. doi:I0.3896/IBRA.I.49.I.I2

Muñoz, I., Henriques, D., Jara, L., Johnston, J. S., ChávezGalarza, J., De La Rúa, P., \& Pinto, M. A. (2017). SNPs selected by information content outperform randomly selected microsatellite loci for delineating genetic identification and introgression in the endangered dark European honey bee (Apis mellifera mellifera). Molecular Ecology Resources, 17(4), 783-795.

Muñoz, I., Henriques, D., Johnston, J. S., Chávez-Galarza, J, Kryger, P., \& Pinto, M. A. (20I5). Reduced SNP panels for genetic identification and introgression analysis in the dark honey bee (Apis mellifera mellifera). PLoS ONE, 10(4)., doi:I0.137//journal.pone.0124365

Parejo, M., Wragg, D., Gauthier, L., Vignal, A., Neumann, P., \& Neuditschko, M. (20|6). Using whole-genome sequence information to foster conservation efforts for the European dark honey bee, Apis mellifera mellifera. Frontiers in Ecology and Evolution, 4, I 40.

Parejo, M., Wragg, D., Henriques, D., Vignal, A., \& Neuditschko, M. (2017). Genome-wide scans between two honey bee populations reveal putative signatures of humanmediated selection. Animal Genetics, 48(6), 704-707. 